\title{
Detailed Review of Ball End Magnetorheological Fluid Finishing Process
}

\author{
ANAND SHARMA ${ }^{1 *}$, ARTI VAISH ${ }^{2}$ AND ANSHU SHARMA ${ }^{3}$ \\ ${ }^{1,2}$ School of Engineering and Technology, ${ }^{3}$ Organizational Behavior and Human Resource \\ Management \\ ${ }^{1,2}$ Ansal University, ${ }^{3}$ O.P. Jindal University \\ ${ }^{1,2}$ Ansal University, Sector -55, Golf Course University, Gurgaon and ${ }^{3}$ Narela Road, Sonepat \\ ${ }^{1,2,3}$ INDIA
}

\begin{abstract}
For precision finishing of various newly and difficult of finish materials like optical glasses, metals, 3D-printed workpieces etc. Ball End Magnetorheological Finishing (BEMRF) finishing processes has been recently developed. This method utilizes a paste like fluid consisting of a base fluid which can be either water or oil, both magnetic and non-magnetic particles and stabilizing agents if necessary. Rheological behavior of this mixture of magnetorheological (MR) fluid with abrasives changes under the influence of magnetic field which in turn regulates the finishing forces during finishing processes. Present study critically reviews the BEMRF process used for achieving nano-level finishing variety of materials like mild steel, EN-31, copper etc. and the factors influenced this process so far which led to further advancements in this method.
\end{abstract}

Keywords: Ball End Magnetorheological Finishing (BEMRF), Rehological, Magnetorheological (MR), Abrasives, Finishing forces

Received: May 16, 2020. Revised: October 2, 2020. Accepted: October 20, 2020. Published: October 31, 2020.

\section{Introduction}

Recently, the demand in most industries like the electronics, optical, aerospace, energy sectors, etc. for the precision surface finished goods like dies, molds, optical glasses, artificial implants etc. has grown rapidly as the properties like, frictional losses, component life under loads, and wear resistance are significantly affected by the quality and magnitude of surface roughness. To achieve a high value of surface finish as well as low finishing cost for new materials having high values of hardness, toughness; strength to weight ratio, etc., advanced finishing technologies have to be implemented. A good surface finish could be obtained from conventional finishing processes like lapping, grinding and honing by selecting optimum machining parameters but these processes possess certain disadvantages like creating burrs, residual stresses, subsurface damage, etc., and also limitations for finishing fragile and 3D intricate shaped materials. However, in order to achieve nonometric level of surface roughness value it is difficult and uneconomical through conventional finishing processes. Various advanced precise surface finishing processes have been developed for finishing different shapes and types of materials by controlling the finishing forces exerted by the finishing tool on the workpiece. Some of the controlled finishing force processes are magnetic float polishing [1], magneto-rheological abrasive flow finishing [2], abrasive flow machining [3], magnetorheological abrasive flow finishing
(MRAFF) [4], magnetorheological jet finishing [5], and magneto-rheological finishing (MRF) [6]. In these processes the finishing forces are controlled by controlling the magnetic flux density by using either a permanent magnet or an electromagnet. However, these processes have limitations in respect to the geometries of the products that can be finished and are capable of finishing limited geometries such as concave, convex, flat, and symmetrically spherical shapes. Ball end magnetorheological finishing (BEMRF) another version of MRF is a novel finishing process based on smart fluids having an ability to control the finishing forces which enables it to finish 3D surfaces to nanometer levels [7]. This process addresses the limitations discussed above with various other non-traditional finishing processes working on the principle of controlling finishing forces developed in the recent past. BEMRF process is capable of finishing a variety of materials, either magnetic materials like diverse alloys of steel, etc. [7], or nonmagnetic materials such as glass [8], silicon [9], aluminum, copper [10], etc.

This article focuses on the design and development of ball end magnetorheological finishing (BEMRF) process, various materials finished so far using this technique and numerous factors affecting the finishing of variety of magnetic or non-magnetic complex shaped materials. 


\section{Ball end magnetorheological finishing (BEMRF)}

This section deals with the design of BEMRF process and the various research work done retailed to this process.

\subsection{Basic Design and Principle}

The limitations of finishing limited geometries such as convex, concave, flat and aspherical due to the constraint in relative movement between workpiece and finishing tool, were surpassed by developing ball end magnetorheological finishing (BEMRF) a variant of MRF process [11]. The first ever setup of BEMRF [12] included vertically oriented MR finishing tool driven by servo motor comprising of cylindrically shaped inner core (made of iron), electromagnet coil and an outer core oriented concentrically to each other (Figure 1). The electromagnet coil is designed to obtain maximum magnetic flux density of $\sim 0.8 \mathrm{~T}$ at the tip of the MR finishing tool. The delivery of MR fluid was controlled by a delivery pump from the storage tank (funnel shape) to the tool tip.

Figure 2(a) represents the direction of magnetic flux flow and formation of a nearly ball shaped highly viscous MR fluid at the tool tip. As soon the MR fluid reaches the tip of the finishing tool, the magnetic CI particles present in the MR fluid aligns themselves along the magnetic field flow direction and forms a chain like structure. The viscosity of the MR fluid can be controlled or varied by controlling the magnetizing current in real time hence controlling the magnetic field strength. The forces exerted by the semi-solid ball end tip comprising of magnetic particles and abrasive grains on the workpiece surface during BEMRF process is shown in figure 2(b).

The strength to hold together the CI particles having cutting edges is provided by the magnetic forces between CI particles. The peaks from the workpiece surface are abraded due to plastic deformation during the rotation of highly viscous ball end tool tip by shearing action of abrasive particles trapped with magnetic CI particles having relative motion with respect to the work surface. The induced magnetic current controls the bonding strength of semi-solid ball end of finishing tool which in turn controls the amount of material to be sheared from the peaks of the workpiece surface by abrasive grains.

According to the microstructure of abrasive and magnetic particles on the workpiece surface as shown in figure $2(b)$, the resultant finishing force $\left(F_{F}\right)$ is a function of normal force $\left(F_{n}\right)$ and shear force $\left(F_{t}\right)$. The normal force is responsible for the depth of penetration in the workpiece surface whereas shear force is responsible for the removal of material.

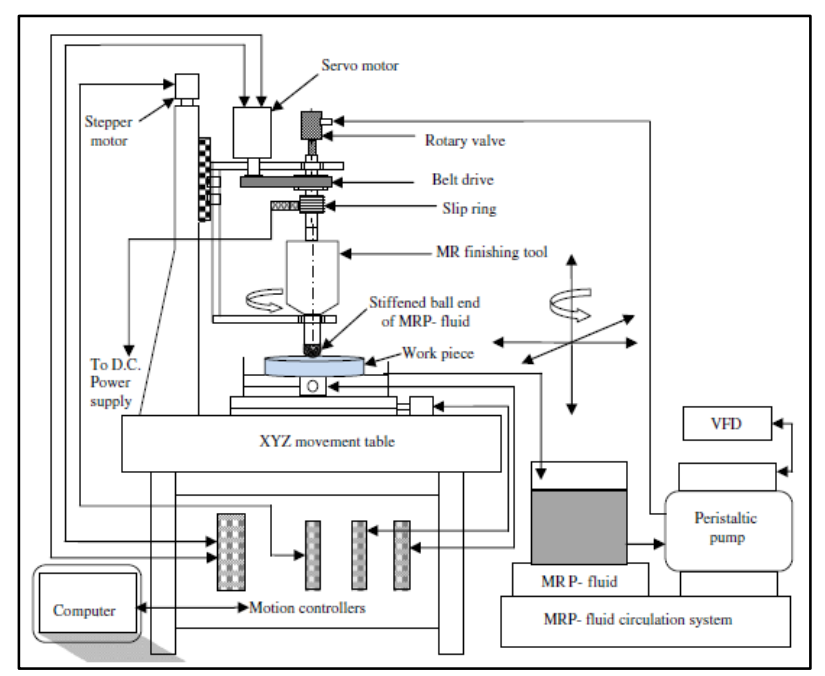

Fig. 1 Schematic of BEMRF process [12]

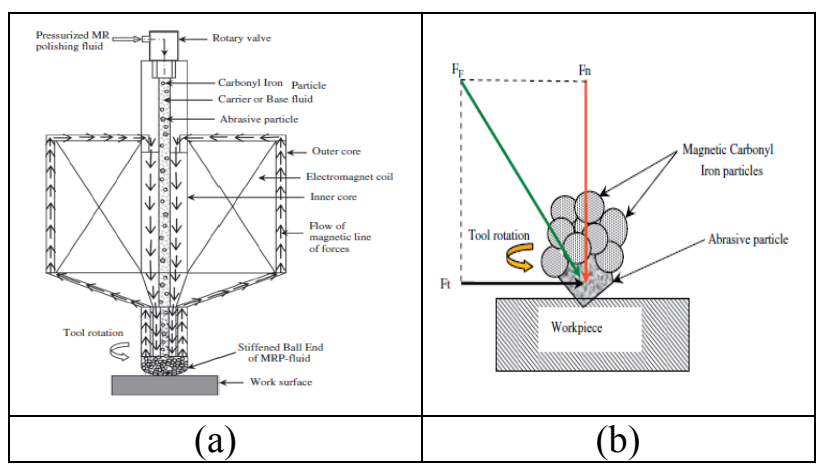

Fig. 2 (a) formation of a semi-solid ball end shape of MR fluid \& magnetic field flow and (b) microstructure of abrasive and magnetic particles on the workpiece surface [12]

\subsubsection{Past Investigations}

From the past study it was found that the original design of the ball end magnetorheological finishing setup did not have a provision of cooling the finishing tool once the current is supplied to the electromagnetic coil. As the finishing tool is in continuous rotation and supply of current the tool gets heated up to quite a large extent which may change the rheological properties of the MR fluid and hinders finishing of the workpiece for longer duration. To eradicate the above mentioned problem, copper cooling coils [6] were wrapped around the electromagnetic coil for continuous cooling as shown in figure 3. The coolant (generally water) is supplied by a chiller maintained at low temperature which helps in maintaining an optimum temperature so as to increase the finishing time.

While carrying out a magnetic simulation over both copper and ferromagnetic material Khan et al. [13] found that in case of copper workpiece the magnetic flux density declined to a great extent and also irregular at the surface while BEMRF process. Being a non-magnetic material (copper) instead of 
two magnetic poles as in case of magnetic materials only one magnetic pole was formed between the tool tip and workpiece surface hence magnetic flux density decreases. Figure $4 a$ \& $4 b$ shows the magnetic flux density distribution for ferromagnetic and copper which clearly indicates that the strength of magnetic flux is high for magnetic materials as compared to the non-magnetic material (copper). Slight improvement in magnetic flux density over copper workpiece surface was observed when supported by mild steel base but significant improvement was obtained by placing a layer of permanent magnet below the copper work-piece. The improvement in magnetic flux density by placing permanent magnet below copper work-piece was experimentally verified by using gauss meter.

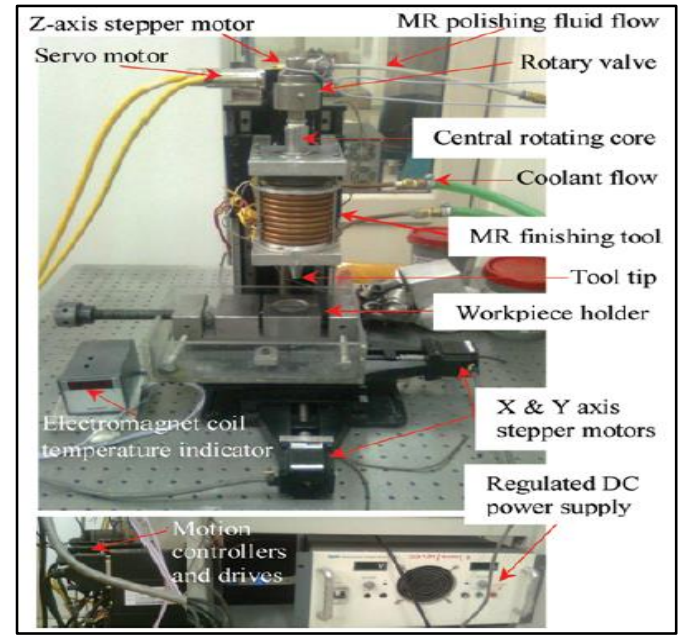

Fig. 3 Copper cooling coils wrapped around the electromagnetic coil [6]

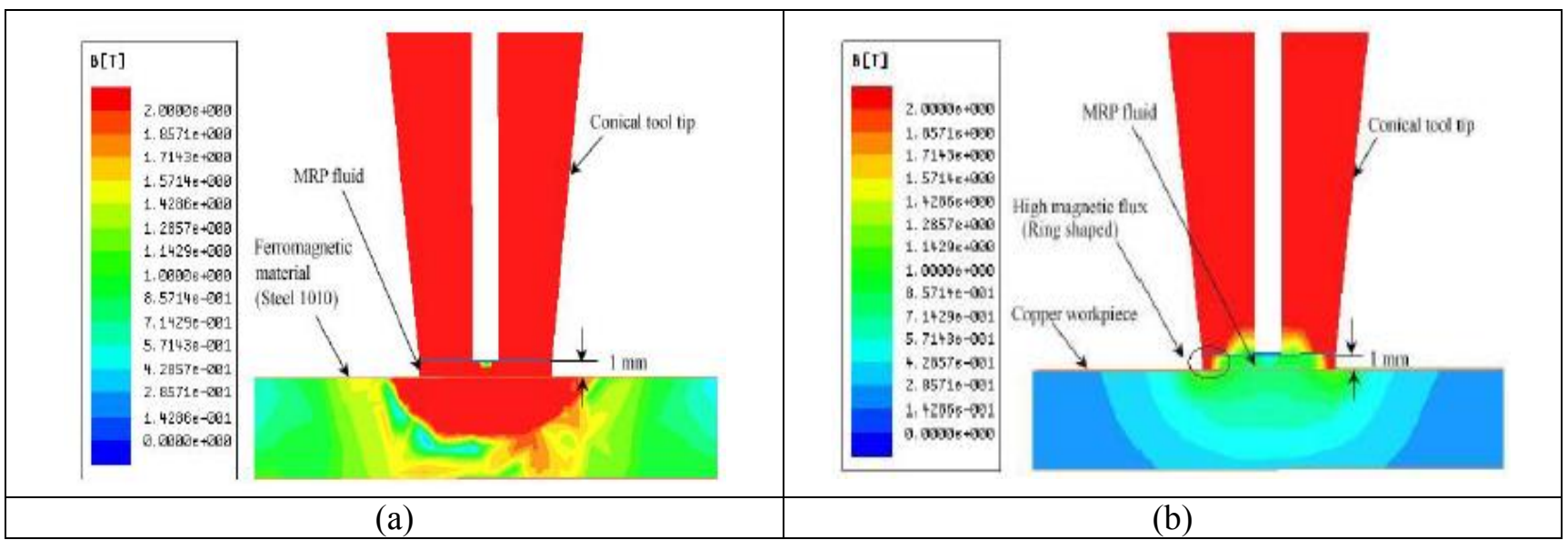

Fig. 4 Magnetic flux density distribution for (a) ferromagnetic material and (b) copper [13]

Further, it was found that due to the central hole inside the rotating core provided for the flow of MR fluid in the setup of BEMRF by Singh et al. [12] the process experienced a non-uniform magnetic flux density at the tool tip which may lead to nonuniformity in surface roughness when used for spot finishing. To generate an unvarying magnetic flux density at the end of a rotating finishing tool tip for providing a uniform surface finish for spot finishing operation of precision components, rotating core having central hole was replaced with solid rotating core tool [14]. A comparative analysis of magnetic flux density was made between finishing of workpiece using rotating core with or without central hole. It was found from the results of the finite element analysis that magnetic flux density at the end surface of a solid rotating core tool was more uniform when compared to the rotating core tool with central hole (Figure 5a \& 5b). Uniform magnetic field at the entire end surface of a solid rotating core tool facilitated the uniform strength of MR polishing fluid at its entire uniformly magnetized end surface. This confirmed that the magnetic normal force has been applied uniformly during the spot nano-finishing on the work-piece surface.

During BEMRF process, the workpiece has to be removed manually and cleaned for calculating surface roughness. In order to automate the BEMRF process, MR fluid which sticks at workpiece surface after the finishing process needs to be removed automatically for automatic measurement of surface roughness. Iqbal et al. [15] made an attempt to automatically clean the workpiece so as to measure the surface roughness for feedback control of the BEMRF process. Automation of the cleaning system for workpiece was achieve in three phases namely design of cleaning strategy, design \& development of cleaning system and automation of cleaning system. To design a cleaning strategy various tests were conducted for cleaning the wokpiece with air spray, water jets, surfactant solutions and kerosene jets. It was found that for the complete removal of MR fluid, kerosene jets were found the best option among all when impinged at various pressures without affecting 


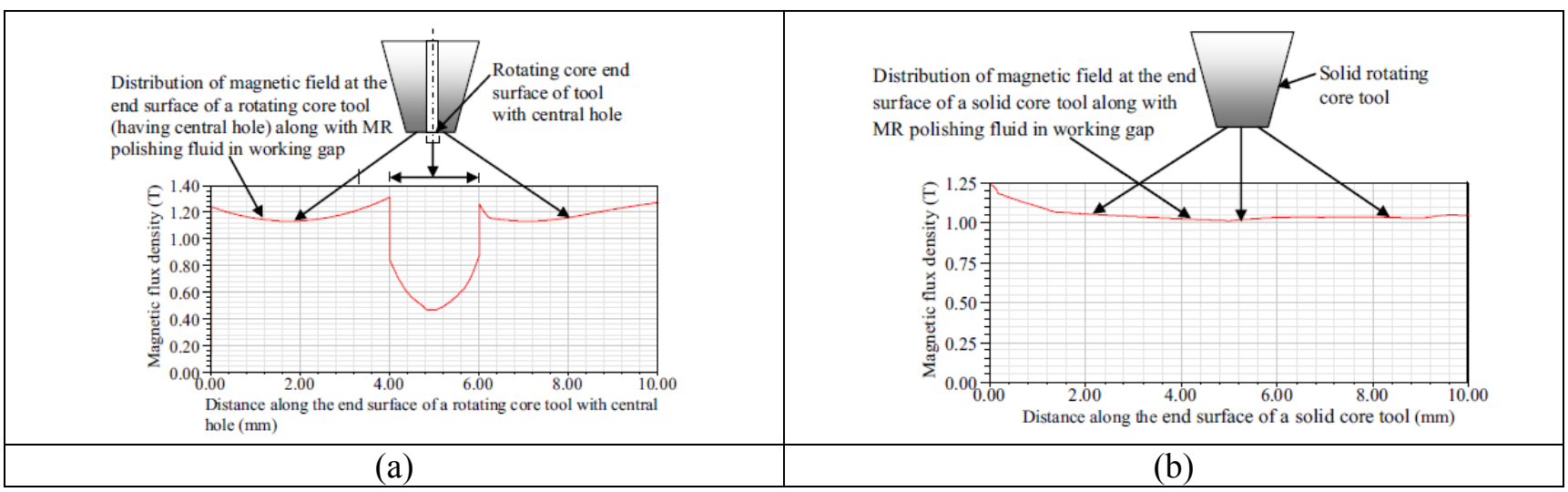

Fig. 5 Distribution of magnetic field at the end surface of (a) rotating core tool with central hole and (b) solid rotating core tool [14]

the surface roughness of the workpiece. The whole cleaning system was automated by the use of electro pneumatic control system to make the system precise, fast and repeatable. Thus an efficient automated cleaning system was developed which aids the feedback control system of BEMRF system.

Recently an attempt has been made to fully automate the BEMRF process [16]. In BEMRF process, magnetically induced normal force can be manually controlled by varying the current supplied to the electromagnet [12]. However, in-process control of finishing forces in BEMRF process requires continuous change of magnetizing current depending upon the inclination of the surface and the roughness profile. In order to completely automate the BEMRF process for finishing of 3D surfaces, a programmable automation controller (PAC) was customized to provide integration of process parameters, motion axes, and in-process magnetizing current (finishing forces). The PAC is interfaced through Visual C\# programming language to develop the graphical user interface (GUI) having all the process parameter controls and a $\mathrm{CNC}$ interface to control motion of the five axes using part programs. Communication between the PAC and the Visual C\# application software is provided by the communication server named ComACRServer [17]. The Visual C\# interface enables the users to import CNC part programs into the GUI and carry out the finishing process with the tool tip following the path defined by the part program. For finishing in automatic mode, magnitude of magnetizing current (with prefix "E") is also included in the part program thereby enabling the control of magnetizing current values within a part program, i.e., different current values in different lines of a single part program. The GUI also contains the controls for the manual operation of the machine. The fully automated fiveaxis CNC BEMRF process was tested on a mild steel workpiece and its result was compared with workpiece finished on conventional three-axis BEMRF machine and five-axis CNC BEMRF machine without in-process current control functionality. With automated five-axis finishing process a uniform surface finish with higher percentage reduction in surface roughness value was obtained when compared to the other two cases.

The literature survey shows that the surface roughness $(\mathrm{Ra})$ is highly influenced by the machining parameter i.e. working gap during BEMRF process. To ensures uniform surface finish throughout the workpiece surface a constant working gap must be maintained during single run. A tapered surface or an error in the linear positioner (Figure $6 a \mathrm{a} 6 \mathrm{~b}$ ) varies the working gap between the tool tip and workpice surface, hence affecting the surface finish. In order to check the tilt or taper error if any in the surface Iqbal et al. [18] scanned the flat surface prior to the finishing process using a chromatic confocal sensor [19]. The height data of peaks and valleys on the workpiece surface are recorded by the confocal sensor and fed to the $\mathrm{z}$ axis motion of the tool tip to maintain a constant gap between the tool tip and the workpiece surface throughout the run. Figure 7 shows a schematic of the control of working gap perpetuation process using a chromatic confocal sensor. Results of the finishing done on flat mild steel workpiece showed that Ra reduced from initial value of $0.831 \mu \mathrm{m}$ to $0.207 \mu \mathrm{m}$ in case of workpiece without tilt error whereas with tilt error the Ra value is reduced from $0.831 \mu \mathrm{m}$ to $0.454 \mu \mathrm{m}$. Despite having tilt error, the Ra reduced from $0.831 \mu \mathrm{m}$ to $0.220 \mu \mathrm{m}$ with perpetuated working gap process. 


\subsection{Material \& Shape of finished workpiece} This section discusses the various materials \& shapes of workpiece finished using ball end magnetorheological finishing (BEMRF), 3-axis CNC BEMRF, 5-axis CNC BEMRF and fully automated 5-axis CNC BEMRF process.

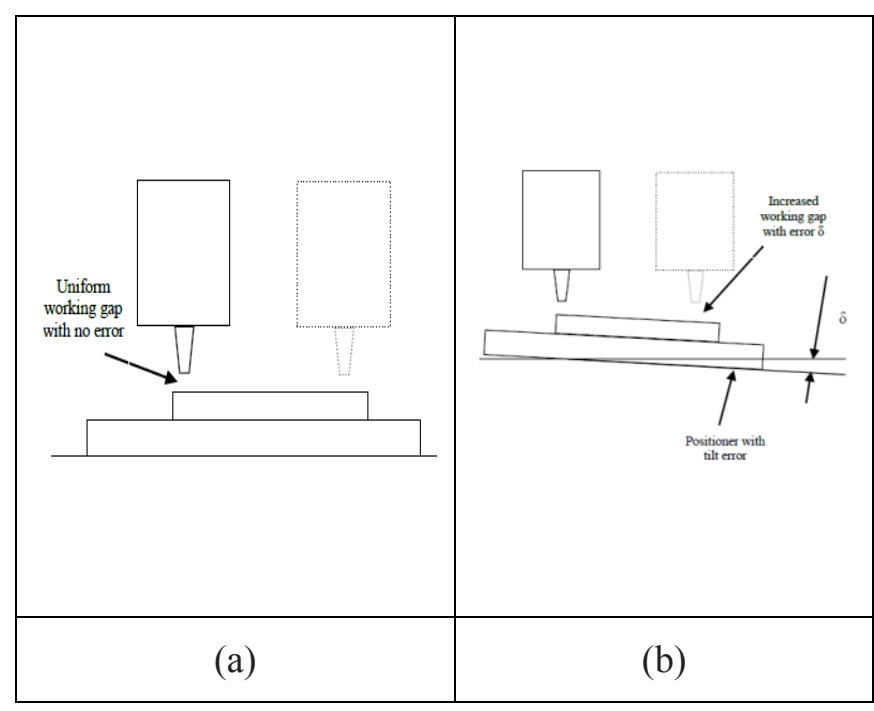

Fig. 6 Working gap (a) with no error and (b) with an error in the linear positioner [18]

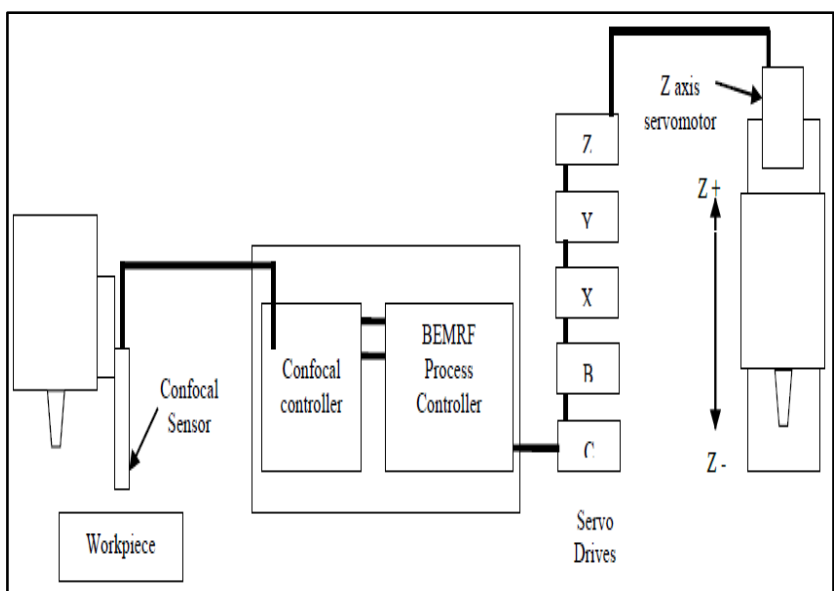

Fig. 7 A schematic of the control of working gap perpetuation process using a chromatic confocal sensor [19]

\subsubsection{Past Investigations}

Singh et al. [6,7,12] designed and developed a computer controlled ball-end magnetorheological (MR) finishing experimental setup to study the process characteristics and performance while finishing flat and 3D surfaces of EN-31 ferromagnetic workpiece and non-magnetic copper workpiece. To visualize the finishing spot shape and size in contact with the workpiece surface the magnetostatic simulations of flux density at the tool tip was carried out. It was found that while keeping the magnetizing current constant the shape and size of the finishing spot in contact with the workpiece surface varies with the variation in working gap. Results showed that the process performance of the finishing process was greatly influenced by magnetic nature of workpiece material, working gap and magnetizing current.

A 3-axis CNC ball end magnetorheological (MR) finishing tool for finishing was developed [6] for finishing 3D work-piece surfaces. The focus was primarily on surface finish and performance evaluation of a typical three-dimensional ferromagnetic work-piece. A 3D workpiece surface was developed by milling process at different angles of projection such as flat, $30^{\circ}, 45^{\circ}$ and curved surface. The effect of number of finishing passes on final surface finish in terms of surface roughness value was also studied. Finite element analysis was done using Iron as a 3D ferromagnetic material to study magnetic flux density distribution between the finishing tool tip and workpiece surface. The results of the experiments performed on a flat ground surface to study the process performance as compared with the milled workpiece surface showed that the surface roughness was reduced as low as $16.6 \mathrm{~nm}, 30.4 \mathrm{~nm}$, $71 \mathrm{~nm}$ and $123.7 \mathrm{~nm}$ on flat, $30^{\circ}, 45^{\circ}$ and curved surface respectively for 60 passes of finishing. The roughness of flat ground surface was reduced as low as $19.7 \mathrm{~nm}$ for $120 \mathrm{~min}$ of finishing. The experimental results demonstrated that the newly developed 3-axis CNC BEMRF process was effective in finishing typical 3D ferromagnetic workpiece surfaces and also for long duration finishing by using cooling coils around the electromagnetic coil.

The effect of finishing time on final surface roughness of rectangular shaped fused silica glass surface after BEMRF process was studied [8]. A total of 5 experiments were conducted on the workpiece surface by increasing the finishing time from $30 \mathrm{~min}$ to $150 \mathrm{~min}$ with an increment of $30 \mathrm{~min}$ in each experiment. Experimental results showed that surface finish improved each time with increase in finishing time of $30 \mathrm{~min}$. Surface roughness decreased to a value of $0.14 \mathrm{~nm}$ from $0.74 \mathrm{~nm}$ after $150 \mathrm{~min}$ of finishing time. It was also found that due to long finishing time, cutting marks on the finished workpiece surface were also visible due to the interaction of abrasives in MR fluid with the surface.

A comparison was made [20] between percentage reduction in surface roughness $\left(\% \Delta \mathrm{R}_{\mathrm{a}}\right)$ calculated by finishing the mild steel work-piece with bidisperse magnetorheological polishing fluid (MRPF) using ball end magnetorheological finishing (BEMRF) tool and $\% \Delta \mathrm{R}_{\mathrm{a}}$ obtained with existing monodispersed MRPF for $30 \mathrm{~min}$. Tool rotational speed, working gap, DC supply, total finishing time, abrasives were 
kept constant whereas carbonyl iron powder of CS and HS grade was used with different combinations for preparing various fluid samples. One sample was prepared for monodisperse MR fluid with $20 \mathrm{vol} \%$ CIP particles (CS grade) and other three samples were synthesized for bidisperse MR fluid having following proportion of CIP particles (18 vol\% CS grade \& 2 vol $\%$ HS grade, 16 vol $\%$ CS grade \& 4 vol $\%$ HS grade and 14 vol $\%$ CS grade \& 6 vol $\%$ HS grade). The magnetorheometery results showed that the maximum yield shear stress and viscosity were observed for bidisperse MRPF (Carbonyl iron powder 16 vol.\% CS grade, 4 vol.\% HS grade, 25 vol.\% $\mathrm{SiC}$ abrasive and 55 vol.\% base fluid) as compared to monodisperse (Carbonyl iron powder 20 vol.\% CS grade, 25 vol.\% SiC abrasive and 55 vol.\% base fluid) MRPF. Percentage reduction in surface roughness was found better by finishing the mild steel workpiece surface using bidisperse MR polishing fluid than monodisperse MRPF. Around $46.25 \%$ improvement in $\% \Delta \mathrm{R}_{\mathrm{a}}$ was observed using bidisperse MR polishing fluid. The characterization of the bidisperse samples were also done [21] under different magnetic field strengths to understand the flow behavior of the newly developed bidisperse MR fluid. After characterization, the experiments were performed with bidisperse MR polishing fluid on mild steel workpiece and percentage reduction in surface roughness $(\% \Delta \mathrm{Ra})$ was calculated and found to be superior.

Saraswathamma et al. [22] experimentally studied the influence of various process parameters viz. core rotational speed, working gap, and magnetizing current on surface finish in terms of percentage reduction in surface roughness of silicon wafer using BEMRF process. MR polishing fluid prepared for finishing of silicon wafer consisted of deionized water as base fluid and cerium oxide as abrasive. Individual effect of process parameters on surface finish in expressions of arithmetical mean roughness (Ra) was studied by applying analysis of variance (ANOVA). The experimental results showed that the working gap was the critical machining parameter for finishing silicon workpiece using BEMRF process. On increasing the working gap the improvement in percent reduction in surface roughness of the silicon workpiece increases. Increase in magnetizing current was found to increase the percentage reduction in $\mathrm{Ra}$ values. Less significant improvement in percentage reduction in $\mathrm{Ra}$ values for different core rotational speeds was observed at lower working gaps. However, at higher working gaps, percentage reduction in $\mathrm{Ra}$ decreases with increase in core rotational speed.
Polishing of polycarbonate material up to a nanometric level surface roughness was done using BEMRF process [13]. Cerium oxide, alumina and diamond abrasives were used to prepare different MR fluid samples for finishing the polycarbonate workpiece. Best result of 54\% reduction in surface roughness was found when finished with diamondbased MR fluid. Almost similar result was found while finishing of the workpiece with alumina based MR fluid. So on the basis of cost of the polishing abrasives, alumina can be used for the polishing of polycarbonate. It was also observed that among all the machining parameters, working gap has the highest impact on the surface finish.

Khan et al. [10] carried out a magnetic simulation over both copper and ferromagnetic material and subsequently copper workpiece was finished by providing a base support of permanent magnet. As discussed above, copper being non-magnetic in nature fails in forming two magnetic poles due to which the magnetic flux density declined to a great extent and also irregular at the surface while BEMRF process resulting in low finishing forces. The magnetic flux density was improved by placing permanent magnet below copper workpiece and experimentally verified. After creating an additional base of permanent magnet while finishing copper workpiece the surface roughness value declined from $35.7 \mathrm{~nm}$ to $7.3 \mathrm{~nm}$ in 30 minutes of finishing time.

Khan et al. [23] also developed an MR fluid with a composition suitable for BEMR finishing of copper. An unusual approach of using permanent magnet as base to create two opposite magnetic pole was followed to enhance the magnetic flux density distribution between the finishing tool tip and copper workpiece surface. The effect of MR fluid composition parameters has been analyzed by the statistical model developed by response surface. Results showed that the copper surface turns black and brown in color on reacting with oxygen due to oxide formation irrespective of the type of carrier fluid. To overcome the problem of change in color of surface, BTA mixed MR fluid has been synthesized for finishing of copper. A novel approach of placing a permanent magnet below the copper workpiece was followed to obtain uniform and high magnetic flux density between the tool tip and workpiece. Using this method, the magnetic flux density thus obtained was enhanced from 0.35 to $0.85 \mathrm{~T}$. Analysis of MR fluid showed the percentage reduction in surface roughness $(\% \Delta \mathrm{Ra})$ firstly increased on increasing the abrasive concentration upto a certain limit and the decreases on further addition of abrasives in the MR fluid. Upon optimization of abrasive and EIP concentration in MR fluid for the copper finishing it 
was found that the amount of abrasives can be maintained at 14 vol \% whereas for EIP 23 vol \% was found optimum. During $30 \mathrm{~min}$ of finishing of copper using optimum MR fluid composition, the surface roughness value was reduced from $65.90 \mathrm{~nm}$ to 38 $\mathrm{nm}$.

Kumar et al. [24] analytically and experimentally studied the influence of MR fluid composition and finishing duration during BEMF of polyactic acid (PLA) workpiece material. The 3D printed PLA workpiece material was built using fused deposition modelling (FDM) process. The workpiece was primarily finished by facing and lapping conventional processes. During preliminary study three types of abrasives namely Alumina $\left(\mathrm{Al}_{2} \mathrm{O}_{3}\right)$ of mesh size 1000, Silicon carbide ( $\mathrm{SiC})$ of mesh size 1000 , and Boron carbide $\left(\mathrm{B}_{4} \mathrm{C}\right)$ of mesh size 1000 were mixed with Electrolytic iron particles (EIPs) and water as base fluid. Alumina $\left(\mathrm{Al}_{2} \mathrm{O}_{3}\right)$ was found suitable for finishing of PLA workpiece material. It was observed from the experimental results that the change in $\% \mathrm{Ra}$ increases up to a certain extent with increase in abrasive concentration in MRP fluid and then starts decreasing due the increase in number of cutting particles in MRP fluid. However, beyond a certain concentration of abrasive in the MRP fluid, $\% \Delta \mathrm{Ra}$ decreases because higher concentration of non-magnetic abrasive particles decreases the magnetic permeability of MRP fluid. Thus hampering the chain formation during the energization process of the MRP fluid resulting in decrease of $\% \Delta R$. From the results, it was noticed that as the EIP concentration increases in the MRP fluid, $\% \Delta \mathrm{Ra}$ increases. This is due to the fact that EIP is the main constituent in the MRP fluid which is responsible for magnetorheological effect and on increasing the EIP concentration the fluid becomes more magnetic in nature and grips the abrasive particles firmly while finishing. An optimum composition of MRP fluid having 16.7 vol\% abrasives, 25 vol\% EIP and 58.83 vol $\%$ of distilled water as base fluid was selected for finishing of PLA workpiece material from the experimental results.

\subsection{Miscellaneous Investigations BEMRF process}

Various other investigations using BEMRF process on different materials discussed above are studied and mentioned under this section. These investigations play a major role in understanding deeply the BEMRF process and other factors influencing this process.

\subsubsection{Past Investigations}

Garg et al. [25] modelled and analyzed the influence of strong magnetic field on flow behavior of MR fluid in BEMRF process using Comsol Multiphysics. The simulation analysis showed that the MR fluid gets stiffened at the tip of the tool and forms almost a hemispherical shape due to the concentration of magnetic flux density, thus providing necessary stiffness for polishing different materials. It was also observed that the intensity of the magnetic field at the tip will depend on the magnetizing current, number of copper turns, magnetic permeability of the MRfluid and iron core.

Singh et al. [26] studied the effect of different mesh size and volume percentage contribution of abrasives in MR fluid on surface roughness of ferromagnetic material using BEMRF process. Silicon carbide was used as abrasives in the MR fluid the mesh size of which was varied from 400 to 1200 and volume percentage contribution ranged from 5 to $25 \mathrm{vol} \%$. MR fluid comprising of $20 \mathrm{vol} \%$ CIP particles, mineral oil as base fluid ranging from 55 to $75 \mathrm{vol} \%$ depending upon the volume percentage of abrasives was synthesized. The results of the BEMRF of ferromagnetic material under specified machining conditions showed that the percentage change in roughness value reduces with increase in abrasive mesh size whereas it decreases with the increase in percentage volume contribution. Optimum composition of MR fluid was analyzed and experiments were conducted accordingly resulting in surface finish value of $82 \mathrm{~nm}$ from an initial value of $214 \mathrm{~nm}$.

Rheological properties of MR fluid specifically field-induced yield stress and shear viscosity were assessed using Casson fluid model [22]. In order to evaluate the behavior of prepared MR fluid for silicon polishing using BEMRF process, a parallelplate magnetorheometer was designed and fabricated. Three different grades of CIP namely CS, OS and HS were used to prepare samples of MR fluid. Ceria-Rhodite grade abrasives along with water as base fluid was used in the MR fluid. Based on the experimental study, it was found that field-induced yield stress of the MR fluid depends directly on the shearing plate surface roughness. The results also showed that the field-induced yield stress and viscosity are a function of CI particle size and flux density.

Niranjan and Jha [27] made an attempt to improve the percentage reduction in surface roughness of mild steel workpiece using MR fluid containing sintered magnetic abrasives. These abrasives were developed by mixing 20 vol\% carbonyl iron powder (CIP) CS grade and $25 \mathrm{vol} \% \mathrm{SiC}$ particles in ball mill. The pellets of $5 \mathrm{~g}$ each made at 8-ton pressure were 
allowed to sinter in tubular furnace maintained at $1200{ }^{\circ} \mathrm{C}$ in the controlled atmosphere of argon. To obtain sintered magnetic abrasives for MR fluid the sintered pellets were crushed in a ball mill. MR fluid thus prepared contained 45 vol\% sintered magnetic abrasives and $55 \mathrm{vol} \%$ of base fluid. It was observed that $\% \Delta \mathrm{Ra}$ increases with increase of tool rotational speed, then reaches a maximum at 600 RPM, and decreases thereafter due to tool aging effect. Hence, optimum tool rotational speed was found as 600 RPM. With the use of sintered magnetic abrasives based MR polishing fluid the tool aging effect has been minimized.

A comparative study based on percent reduction in surface roughness $(\% \Delta R a)$ on mild steel surface with synthesised and unbounded magnetic abrasives based MRP fluid was carried out [28]. The most significant machining parameter affecting $\% \Delta R a$ was observed to be the gap between the workpiece surface and finishing tool tip i.e. working gap. Additionally, magnetising current and tool rotational speed also provides secondary contribution to $\% \Delta R a$. The response $\% \Delta R a$ decreases continuously with the increase of working gap and the response increases with increase of magnetizing current. $\% \Delta R a$ first increases by increasing the tool rotational speed and reach at its maximum value, thereafter decreases due to tool aging. Therefore, an optimum tool rotational speed was found as $500 \mathrm{rpm}$. Predicted response $\% \Delta R a$ was calculated and found as 54.69 by regression model at an optimum machining conditions of magnetizing current, tool rotational speed and working gap.

Theoretical investigations based on modeling of surface roughness and mechanism of material removal followed with experimental verification on mild steel workpiece using BEMRF process were carried out by Alam \& Jha [29]. The study of microstructure of the constituents of the MRP fluid had been proposed for better understanding of intricate structure of magnetically controlled MR fluid during finishing. Based on theoretical modelling of normal and shear forces acting on the abrasive induced magnetically, the material removal process and wear behavior during BEMRF finishing had been analyzed. Experimental value of surface roughness was compared to the theoretical value of surface roughness computed using a mathematical model developed to evaluate the roughness. A close agreement of theoretical and experimental results was observed at low values of magnetizing current which validates the proposed model. $7.23 \%$ to $31.19 \%$ of error between the experimental and theoretical values was found. Occurrence of this error was due to the reason that the model developed theoretical for predicting the surface roughness took into account only the axial variation of the magnetic flux density neglecting the variations radially in the working gap. However, variation of the flux density also occurs radially from the centre of the tool to the periphery of the tool which leads to the variation in theoretical and experimental values of surface roughness.

Iqbal and Jha [30] established finishing parameter sets in terms of reduction in surface roughness for the surfaces to be finished using BEMRF process based on finishing time. Optimum finishing parameter sets were found providing an optimum time based finishing, an algorithm for parameter selection was developed to select the next best set of finishing parameters to accomplish maximum reduction in surface roughness in the next finishing cycle. The operation of BEMRF process was controlled automatically using a predefined numerical control part program. After every finishing cycle measurement of surface roughness was done using Confocal sensor which acted as an initial roughness value for next finishing cycle. Closed loop implementation of BEMRF process was carried out using the NC part program and developed algorithm. It was found that implementation of closed loop BEMRF process provided optimum time for roughness reduction while making the process automatic. Experimental results showed on fully automating the process surface roughness value was brought down to a range of $60 \mathrm{~nm}$ in 200 minutes of finishing time from initial $800 \mathrm{~nm}$ range. The same reduction in surface roughness was achieved in a finishing time of $360 \mathrm{~min}$ by using individual parameter set.

A finishing time based reduction in surface roughness using BEMRF process study was carried out during finishing of EN-31 steel [31]. Optimization of the machining parameters, viz. magnetizing current, working gap and spindle speed was done using 3-factor central composite design technique. Finishing cycle of $40 \mathrm{~min}$ was fixed for each selected set of parameters and surface roughness was measured after each finishing cycle. Transient surface roughness reduction phenomenon was established in BEMRF process based on the results found. It was also observed that the surface roughness reduction over a period of time is a gradually decreasing phenomenon i.e. certain set of finishing parameters will become ineffective and uneconomical with time.

Alam et al. [32] studied the composition effects of polishing fluid on finishing forces i.e. normal and shear forces. Vol.\% of magnetic and non-magnetic abrasives were varied from 5 to $25 \%$ and 5 to $20 \%$ 
respectively forming different samples of MR polishing fluid. Experimental results while finishing of mild steel using BEMRF process showed that with increase in magnetic CIPs concentration in MR fluid both normal and shear forces increased. This is due to the reason that higher vol\% of CIPs increases the magnetic permeability of the MR fluid increasing the viscosity when electrically energized. While increasing the concentration of non-magnetic abrasives in MR fluid, it was observed that the magnitude of forces initially increased with increase in amount of non-magnetic abrasives and then starts to drop after a certain point. This may be because initially with increase in concentration of abrasives voids between the CIPs chains were filled by the abrasives thus strengthening the chain structure of the MR fluid. However, past a certain limit of abrasives it starts hindering the chain formation resulting in large number of broken chains leading to decrease in magnitude of forces during finishing.

\section{CONCLUSION}

Critical review of BEMRF high precision finishing techniques is presented in this article. Following conclusions can be drawn from the above discussion.

- The BEMRF process is an effective finishing process for finishing ferromagnetic as well as non-magnetic materials of different shapes and sizes. Surface roughness up to nanometer level is achieved without subsurface damage.

- High demand for nano-finished and accurate components gave rise to the automation of the BEMRF process. Almost all phases of the process have been automated, still an effort in placing and retrieving the workpiece from the die can be made.

- The present study state that BEMRF process is used to obtain very high level surface finish on variety of workpiece materials. It is observed that magnetic field intensity has the highest contribution on the yield stress and viscosity of the MR fluid.

- The bimodal MR polishing fluids can improve the surface finish of various materials. However, not much of research has been done in the field of finishing of nonmagnetic materials using bidisperse MR fluids.

- Though most of the research is focused on finishing of metals or non-metals using BEMRF process very scarce study has been done for finishing of composite materials.
- Closed loop finishing of BEMRF process can be carried out for different materials.

\section{References:}

[1] Maan, S., Singh, G., \& Singh, A. K., Nanosurface-finishing of permanent mold punch using magnetorheological fluid-based finishing processes, Materials and Manufacturing Processes, Vol. 32, No. 9, 2017, pp. 1004-1010.

[2] Nagdeve, L., Jain, V. K., \& Ramkumar, J., Differential finishing of freeform surfaces (knee joint) using R-MRAFF process and negative replica of workpiece as a fixture, Machining Science and Technology, Vol. 22, No. 4, 2018, pp. 671-695.

[3] Sambharia, J., \& Mali, H. S., Characterization and Optimization of Rheological Parameters of Polymer Abrasive Gel for Abrasive Flow Machining, J. Mater. Sci. Surf. Eng, Vol. 5, No. 3, 2017, pp. 549-555.

[4] Jha, S., \& Jain, V. K., Design and development of the magnetorheological abrasive flow finishing (MRAFF) process, International Journal of Machine Tools and Manufacture, Vol. 44, No. 10, 2004, pp. 1019-1029.

[5] Kordonski, W., \& Shorey, A., Magnetorheological (MR) jet ${ }^{\mathrm{TM}}$ finishing technology, In Electrorheological Fluids and Magnetorheological Suspensions, 2007, pp. 340-346.

[6] Singh, A. K., Jha, S., \& Pandey, P. M., Nanofinishing of a typical 3D ferromagnetic workpiece using ball end magnetorheological finishing process, International Journal of Machine Tools and Manufacture, Vol. 63, 2012, pp. 21-31.

[7] Singh, A. K., Jha, S., \& Pandey, P. M., Magnetorheological ball end finishing process, Materials and Manufacturing Processes, Vol. 27, No. 4, 2012, pp. 389-394.

[8] Singh, A. K., Jha, S., \& Pandey, P. M., Nanofinishing of fused silica glass using ballend magnetorheological finishing tool, Materials and Manufacturing Processes, Vol. 27, No. 10, 2012, pp. 11391144.

[9] Saraswathamma, K., Jha, S., \& Rao, P. V., Experimental investigation into ball end magnetorheological finishing of silicon, Precision Engineering, Vol. 42, 2015, pp. 218-223.

[10] Khan, D. A., Alam, Z., \& Jha, S., Nanofinishing of copper using ball end magnetorheological finishing (BEMRF) process, In ASME 2016 International Mechanical Engineering 
Congress and Exposition, 2016, pp. V002T02A002-V002T02A002.

[11] Kordonski, W. I., \& Jacobs, S. D., Magnetorheological finishing, International Journal of modern physics B, Vol. 10, No. 23 and 24, 1996, pp. 2837-2848.

[12] Singh, A. K., Jha, S., \& Pandey, P. M., Design and development of nanofinishing process for 3D surfaces using ball end MR finishing tool, International Journal of Machine Tools and Manufacture, Vol. 51, No. 2, 2011, pp. 142-151.

[13] Khan, D. A., Kumar, J., \& Jha, S., Magnetorheological nano-finishing of polycarbonate, International Journal of Precision Technology, Vol. 6, No. 2, 2016, pp. 89-100.

[14] Khurana, A., Singh, A. K., \& Bedi, T. S., Spot nanofinishing using ball nose magnetorheological solid rotating core tool, The International Journal of Advanced Manufacturing Technology, Vol. 92, No. (1-4), 2017, pp. 1173-1183.

[15] Iqbal, F., Rammohan, R., Patel, H. A., \& Jha, S., Design and development of automated workpiece cleaning system for ball end magneto-rheological finishing process, In International Conference on Advances in Materials \& Manufacturing, 2016, pp. 289-295.

[16] Alam, Z., Iqbal, F., Ganesan, S., \& Jha, S., Nanofinishing of 3D surfaces by automated five-axis $\mathrm{CNC}$ ball end magnetorheological finishing machine using customized controller, The International Journal of Advanced Manufacturing Technology, Vol. 100, No. (5-8), 2019, pp. 1031-1042.

[17] Parker Hannifin (2009) ComACRServer6 user's guide.

http://divapps.parker.com/divapps/emn/pdf/AC R/ComACRServer6_User_Guide.pdf. Accessed 9 June 2016.

[18] Iqbal, F., Alam, Z., Khan, D. A., \& Jha, S., Constant work gap perpetuation in ball end magnetorheological finishing process, International Journal of Precision Technology, Vol. 8, No. (2-4), 2019, pp. 397410.

[19] Luo, D., Kuang, C., \& Liu, X., Fiber-based chromatic confocal microscope with Gaussian fitting method, Optics \& Laser Technology, Vol. 44, No. 4, 2012, pp. 788-793.

[20] Niranjan, M., Jha, S., \& Kotnala, R. K., Ball end magnetorheological finishing using bidisperse magnetorheological polishing fluid, Materials and Manufacturing Processes, Vol. 29, No. 4, 2014, pp. 487-492.
[21] Niranjan, M. S., \& Jha, S., Flow behaviour of bidisperse MR polishing fluid and ball end MR finishing, Procedia Materials Science, Vol. 6, 2014, pp. 798-804.

[22] Saraswathamma, K., Jha, S., \& Rao, P. V., Rheological characterization of MR polishing fluid used for silicon polishing in BEMRF process, Materials and Manufacturing Processes, Vol. 30, No. 5, 2015, pp. 661-668.

[23] Khan, D. A., \& Jha, S., Synthesis of polishing fluid and novel approach for nanofinishing of copper using ball-end magnetorheological finishing process, Materials and Manufacturing Processes, Vol.33, No. 11, 2018, pp. 11501159.

[24] Kumar, A., Alam, Z., Khan, D. A., \& Jha, S., Nanofinishing of FDM-fabricated components using ball end magnetorheological finishing process, Materials and Manufacturing Processes, Vol. 34, No. 2, 2019, pp. 232-242.

[25] Garg, H., Negi, V. S., Kharola, A. S., \& Sharma, R., Effect of Magnetic Field on MR-Fluid in Ball End Magnetorheological Finishing, In COMSOL Conference, 2014.

[26] Singh, A. K., Jha, S., \& Pandey, P. M., Performance analysis of ball end magnetorheological finishing process with MR polishing fluid, Materials and Manufacturing Processes, Vol. 30, No. 12, 2015, pp. 14821489.

[27] Niranjan, M. S., \& Jha, S., Experimental investigation into tool aging effect in ball end magnetorheological finishing, The International Journal of Advanced Manufacturing Technology, Vol. 80, No. (9-12), 2015, pp. 1895-1902.

[28] Niranjan, M. S., \& Jha, S., Optimum selection of machining parameters in ball end magnetorheological finishing process, International Journal of Precision Technology, Vol. 5, No. (3-4), 2015, pp. 217-228.

[29] Alam, Z., \& Jha, S., Modeling of surface roughness in ball end magnetorheological finishing (BEMRF) process, Wear, Vol.374, 2017, pp. 54-62. 
[30] Iqbal, F., \& Jha, S., Closed Loop Ball End Magnetorheological Finishing Using In-situ Roughness Metrology, Experimental Techniques, Vol. 42, No. 6, 2018, pp. 659-669.

[31] Iqbal, F., \& Jha, S., Experimental investigations into transient roughness reduction in ball-end magneto-rheological finishing process, Materials and Manufacturing Processes, Vol. 34, No. 2, 2019, pp. 224-231.

[32] Alam, Z., Khan, D. A., Iqbal, F., \& Jha, S., Effect of polishing fluid composition on forces in ball end magnetorheological finishing process, International Journal of Precision Technology, Vol. 8, No. (2-4), 2019, pp. 365378.

\section{Creative Commons Attribution License 4.0} (Attribution 4.0 International, CC BY 4.0)

This article is published under the terms of the Creative Commons Attribution License 4.0

https://creativecommons.org/licenses/by/4.0/deed.en_US 